\title{
Theory vs. Practice in the Design and Analysis of Algorithms
}

\author{
Robert E. Tarjan \\ Department of Computer Science, Princeton University and HP Labs
}

\begin{abstract}
In this talk I'll explore gaps between the theoretical study of algorithms and the use of algorithms in practice. Examples will be drawn from my own experiences in industry and academia, and will include data structures and network algorithms. Based on these examples I'll try to draw conclusions to help guide the work of theoreticians and experimentalists, in an effort to make this work more relevant to the needs of practitioners.
\end{abstract}

